\title{
EXACTNESS OF THE DOUBLE DUAL
}

\author{
R. R. COLBY AND K. R. FULLER ${ }^{1}$
}

\begin{abstract}
Let $\boldsymbol{R}$ be an artinian ring. The double $\boldsymbol{R}$-dual ( )** preserves monomorphisms, is left exact, or is right exact if and only if $R$ is QF-3, has dominant dimension at least 2, or is QF, respectively. Furthermore, both double dual functors preserve epimorphisms of finitely generated (left and right) modules if and only if both dual functors ( )* are exact on short exact sequences of finitely generated torsionless modules, and these conditions are equivalent to finitely generated torsionless modules being reflexive.
\end{abstract}

A one-sided noetherian ring is QF, i.e. two-sided artinian and self-injective, if and only if either of the contravariant left exact $R$-dual functors ()$^{*}=\operatorname{Hom}(, R)$ : $R$-Mod $\rightarrow$ Mod- $R$ or ( $)^{*}:$ Mod- $R \rightarrow R$-Mod is actually exact (see [3]) and then, of course, the two composites ( )** of these dual functors are exact on $R$-Mod and Mod- $R$, respectively. A ring $R$ has left dominant dimension at least $n$, written $\operatorname{dom} \operatorname{dim}_{R} R \geqslant n$, in case the first $n$ terms in the minimal injective resolution of ${ }_{R} R$ are projective, and the left and right dominant dimensions of a one-sided artinian ring are equal by [6, Theorem 1.1] and [9, Theorem 7.7] (see also [2], [5], [7]).

Rings of dominant dimension at least one, and at least two, as well as QF rings, have been extensively studied. (See [9] for a thorough account.) A left or right artinian ring $R$ is (left) QF-3 in the sense that it has a minimal faithful (left) module if and only if $\operatorname{dom} \operatorname{dim} R \geqslant 1$; and $\operatorname{dom} \operatorname{dim} R>2$ if and only if $R$ is QF-3 and is its own maximal quotient ring. Although it has apparently not been noticed before, all three of these kinds of rings can be characterized by exactness of the double dual. Indeed, we shall prove here that a left or right artinian ring $R$ satisfies dom $\operatorname{dim} R \geqslant 1$ or $\operatorname{dom} \operatorname{dim} R \geqslant 2$ if and only if ( )** preserves monomorphisms in $R$-Mod or is left exact on $R$-mod, respectively. Furthermore, if $R$ is right noetherian or left artinian, then ( $)^{* *}$ is right exact on $R$-mod if and only if $R$ is QF. In addition we shall show that if $R$ is noetherian, both double dual functors preserve epimorphisms of finitely generated (left and right) modules if and only if both dual functors are exact on short exact sequences of finitely generated torsionless modules, and these conditions are equivalent to finitely generated torsionless modules being reflexive.

Received by the editors March 25, 1980.

1980 Mathematics Subject Classification. Primary 16A35, 16A36.

'Fuller's research was partially supported by National Science Foundation Grant MCS 77-00431 A01, and was carried out while he was a Visiting Professor at the University of Hawaii. He gratefully acknowledges the support of both institutions. 
We begin by recalling that a left or right artinian ring $R$ is QF-3 if and only if there are idempotents $e, f \in R$ such that $R e$ and $f R$ are faithful and the functors $\operatorname{Hom}_{f R f}(, f R e)$ and $\operatorname{Hom}_{e R e}(, f R e)$ define a duality between the categories of finitely generated left $f R f$ - and right $e R e$-modules. In this case $R e$ and $f R$ are injective over $R$ and finitely generated over $e R e$ and $f R f$, respectively, $\operatorname{Hom}_{f R f}(f R, f R e) \cong R e, \operatorname{Hom}_{e R e}(R e, f R e) \cong f R$ and $f R e$ is an injective cogenerator over both $f R f$ and $e R e$. (See [2], [3], [6].) Also we recall that for any ring $R$ there is a natural transformation $\sigma: 1_{R-M o d} \rightarrow()^{* *}$, defined via the usual evaluation maps $\sigma_{M}$ : $M \rightarrow M^{* *}$, and $\sigma_{P}$ is an isomorphism for any finitely generated projective $R$-module $P$. An $R$-module $M$ is called torsionless (reflexive) in case $\sigma_{M}$ is a monomorphism (an isomorphism); and it is well known (e.g. see [6]) that $M^{*}$ is always torsionless. Now we are ready to prove our first two results.

THEOREM 1. Let $R$ be a left or right artinian ring. Then ( $)^{* *}$ preserves monomorphisms in $R$-Mod if and only if $R$ is $Q F-3$.

Proof. $(\Rightarrow)$ Consider the commuting diagram of left modules

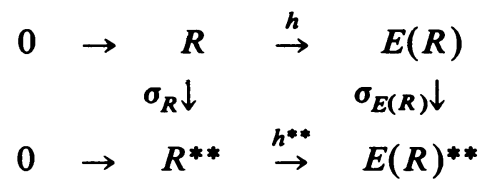

where $E(R)$ denotes the injective envelope of ${ }_{R} R$. Suppose that $h^{* *}$, as well as the inclusion map $h$, is monic. Then $\operatorname{Ker} \sigma_{E(R)} \cap \operatorname{Im} h=0$ so, since $\operatorname{Im} h$ is essential in $E(R), \sigma_{E(R)}$ is monic and hence $E(R)$ is torsionless. If $R$ is left artinian, then $E(R)$ is finitely cogenerated and if $R$ is right artinian then direct products of projective left $R$-modules are projective [1]. Thus, in either event, $R$ is QF-3.

$(\Leftarrow)$ Suppose that $R$ is QF-3 with minimal faithful left and right modules $R e$ and $f R$, where $e$ and $f$ are idempotents in $R$. Then there are natural isomorphisms

$$
\begin{aligned}
\operatorname{Hom}_{f R f}\left(f R \otimes_{R} X, f R e\right) & \cong \operatorname{Hom}_{R}\left(X, \operatorname{Hom}_{f R f}(f R, f R e)\right) \\
& \cong \operatorname{Hom}_{R}(X, R e) \\
& \cong \operatorname{Hom}_{R}(X, R) \otimes R e=X^{*} \otimes R e
\end{aligned}
$$

for $X \in R$-Mod, so that

$$
\operatorname{Hom}_{f R f}(f R \otimes, f R e) \cong \operatorname{Hom}_{R}(, R e) \cong()^{*} \otimes R e
$$

as contravariant functors from $R$-Mod to Mod-eRe. Since ${ }_{f R f} f R$ is a cogenerator and $R e$ is a faithful left ideal, we see from the first isomorphism that

$$
f R \otimes X=0 \text { if and only if } X^{*}=0,
$$

and similarly for a right module $Y$

$Y \otimes R e=0$ if and only if $Y^{*}=0$.

From the second isomorphism, since $R e$ is injective, we see that

$$
()^{*} \otimes R e: R-M o d ~ \rightarrow \text { Mod-eRe }
$$


is an exact functor. Now suppose that $0 \rightarrow K \rightarrow M$ is exact in $R$-Mod, and let $M^{*} \rightarrow K^{*} \rightarrow Y \rightarrow 0$ be exact in Mod- $R$. Then both

$$
M^{*} \otimes R e \rightarrow K^{*} \otimes R e \rightarrow 0
$$

and

$$
M^{*} \otimes R e \rightarrow K^{*} \otimes R e \rightarrow Y \otimes R e \rightarrow 0
$$

are exact in Mod-eRe, so $Y \otimes R e=0$ and hence $Y^{*}=0$. But then $0 \rightarrow K^{* *} \rightarrow$ $M^{* *}$ is exact.

TheOREM 2. Let $R$ be left or right artinian. Then ( )** is left exact on $R$-Mod if and only if $\operatorname{dom} \operatorname{dim} R \geqslant 2$.

Proof. $(\Rightarrow)$ Let

$$
0 \rightarrow \rightarrow_{R} R \stackrel{h_{0}}{\rightarrow} E_{1} \stackrel{h_{1}}{\rightarrow} E_{2} \rightarrow \cdots
$$

be the minimal injective resolution of ${ }_{R} R$ and assume that ( )** is left exact on $R$-Mod. Then by Theorem $1, E_{1}$ is finitely generated and projective so in the commutative diagram with exact rows

$$
\begin{array}{ccccccc}
0 & \rightarrow & R & \stackrel{h_{0}}{\rightarrow} & E_{1} & \stackrel{h_{1}}{\rightarrow} & E_{2} \\
& & \sigma_{0} \downarrow & & \sigma_{1} \downarrow & & \sigma_{2} \downarrow \\
0 & \rightarrow & R^{* *} & \stackrel{h_{0}^{* *}}{\rightarrow} & E_{1}^{* *} & \stackrel{h_{1}^{* *}}{\rightarrow} & E_{2}^{* *}
\end{array}
$$

$\sigma_{0}$ and $\sigma_{1}$ are isomorphisms. It easily follows that $\operatorname{Im} h_{1} \cap \operatorname{Ker} \sigma_{2}=0$, so $E_{2}=$ $E\left(\operatorname{Im} h_{1}\right)$ is torsionless and hence projective.

$(\Leftarrow)$ Suppose that $0 \rightarrow R_{R} \rightarrow E_{1} \rightarrow E_{2}$ is exact in Mod-R with $E_{1}$ and $E_{2}$ injective and projective. Let

$$
0 \rightarrow K \stackrel{h}{\rightarrow} M \stackrel{g}{\rightarrow} N \rightarrow 0
$$

be exact in $R$-Mod, and let $0 \rightarrow N^{*} \rightarrow M^{*} \rightarrow K^{*} \rightarrow Y \rightarrow 0$ be exact in Mod- $R$. Then, since $R$ is QF-3, ( )** preserves monomorphisms by Theorem 1, and we see from the exact sequences

$$
0 \rightarrow Y^{*} \rightarrow K^{* *} \rightarrow M^{* *} \text { and } 0 \rightarrow K^{* *} \rightarrow M^{* *}
$$

that $Y^{*}=0$. But then, since the $E_{i}$ are projective, we also have

$$
\operatorname{Hom}_{R}\left(Y, E_{i}\right)=0 \quad(i=1,2) .
$$

From this observation, the injectivity of the $E_{i}$, and the left exactness of Hom, it follows that the last two rows and the columns are exact in the commutative diagram

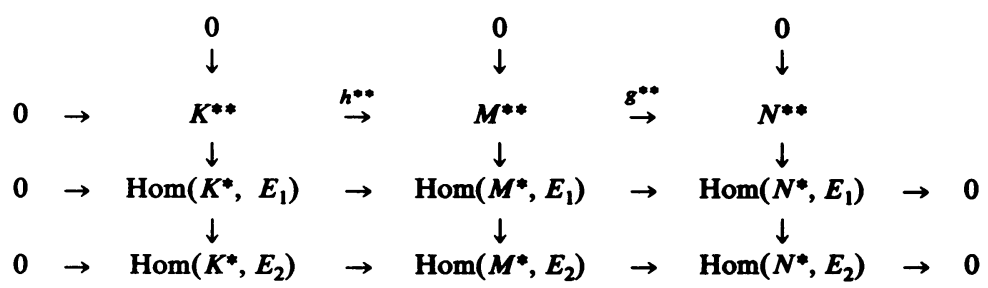


So, since $h^{* *}$ is monic by Theorem 1 , it only remains to show that $\operatorname{Im} h^{* *}=$ $\operatorname{Ker} g^{* *}$. But this follows from ordinary diagram chasing.

Suppose now that ( $)^{* *}$ is right exact on $R$-Mod. Then we see from the 5-Lemma and a commutative diagram

$$
\begin{array}{cccccccc}
P_{1} & \rightarrow & P_{0} & \rightarrow & M & \rightarrow & 0 \\
\sigma_{1} \downarrow & & \sigma_{0} \downarrow & & \sigma_{M} \downarrow & & \\
P_{1}^{* *} & \rightarrow & P_{0}^{* *} & \rightarrow & M^{* *} & \rightarrow & 0
\end{array}
$$

that every finitely presented left $\boldsymbol{R}$-module is reflexive (and so torsionless). Thus we can easily prove

THEOREM 3. Let $R$ be left artinian or right noetherian. Then ()$^{* *}$ is right exact on $R$-Mod if and only if $R$ is $Q F$.

Proof. Let ( ) ${ }^{* *}$ be right exact on $R$-Mod. If $I$ is a finitely generated left ideal of $R$ then, as we saw previously, $R / I$ is torsionless. It follows that $l(r(I))=I$. But then a chain $I_{1}>I_{2}>\ldots$ of finitely generated left ideals yields $r\left(I_{1}\right)<r\left(I_{2}\right)$ $<\ldots$ Thus if $R$ is right noetherian, $R$ has d.c.c. on principal left ideals and hence is right artinian (see [0, p. 321]); now $R$ also has a.c.c. on finitely generated left ideals, so $R$ is left artinian. If every finitely generated left module over a left artinian ring is torsionless, then each indecomposable injective left $R$-module embeds in $R$ because all of its finitely generated submodules do, so $R$ is QF (cf. [8]).

Our final theorem gives several characterizations of those noetherian rings for which the functors ()$^{* *}$ preserve epimorphisms of both finitely generated left and right modules. We suspect that the conditions of this theorem actually do imply that ( )** preserves all epimorphisms, at least for artinian rings.

THEOREM 4. If $R$ is a noetherian ring, then the following are equivalent.

(a) The functors ( $)^{* *}$ preserve epimorphisms of finitely generated left and right $R$-modules.

(b) $\sigma_{M}$ is an epimorphism for every left and every right finitely generated $R$-module M.

(c) Finitely generated torsionless left and right $R$-modules are reflexive.

(d) The functors ( )* are exact on short exact sequences of left and of right finitely generated torsionless $\boldsymbol{R}$-modules.

Proof. (a) $\Rightarrow(b)$. Since $\sigma_{P}$ is an isomorphism for finitely generated projective $P$, this implication follows from the diagram

$$
\begin{array}{ccccc}
P & \rightarrow & M & \rightarrow & 0 \\
\sigma_{P} \downarrow & & \sigma_{M} \downarrow & & \\
P^{* *} & \rightarrow & M^{* *} & \rightarrow & 0
\end{array}
$$

(b) $\Rightarrow$ (c). This is clear.

(c) $\Rightarrow$ (d). First observe that, since $R$ is noetherian, duals of finitely generated modules are finitely generated. Then, assuming (c), let

$$
0 \rightarrow A \stackrel{f}{\rightarrow} B \stackrel{g}{\rightarrow} C \rightarrow 0
$$


be an exact sequence of finitely generated torsionless left $\boldsymbol{R}$-modules. It will suffice to show that $f^{*}: B^{*} \rightarrow A^{*}$ is an epimorphism. Applying ( $)^{*}$ and letting $D=$ Coker $g^{*} \cong \operatorname{Im} f^{*}$ we obtain a commutative diagram of right $R$-modules

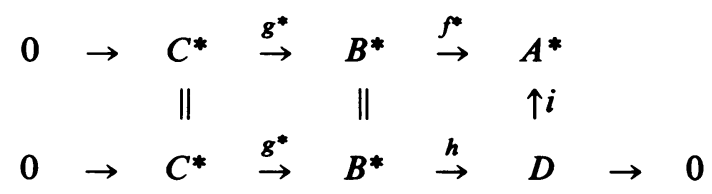

with exact rows in which $i$ is a monomorphism. Applying ( $)^{*}$ and $\sigma$ we get another commutative diagram, also with exact rows

$$
\begin{array}{ccccccccc}
0 & \rightarrow & A & \rightarrow & B & \rightarrow & C & \rightarrow & 0 \\
& & \sigma_{A} \downarrow & & \sigma_{B} \downarrow & & \sigma_{C} \downarrow & & \\
0 & \rightarrow & A^{* *} & \rightarrow & B^{* *} & \rightarrow & C^{* *} & \rightarrow & 0 \\
& & i^{*} \downarrow & & \| & & \| & & \\
0 & \rightarrow & D^{*} & \stackrel{h^{*}}{\rightarrow} & B^{* *} & \rightarrow & C^{* *} & \rightarrow & 0
\end{array}
$$

Since $\sigma_{B}$ and $\sigma_{C}$ are isomorphisms, it follows from the 5-Lemma that $i^{*} \circ \sigma_{A}$ is an isomorphism. Thus, since $A$ is reflexive, $i^{*}$ is an isomorphism. Now considering the diagram

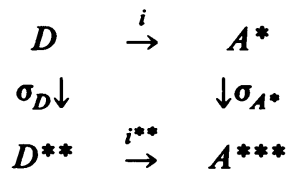

we see that $i$ is an isomorphism and hence $f^{*}$ is epic as desired. (This proof closely parallels one of Morita as presented in [0, pp. 278-279].)

(d) $\Rightarrow$ (a). An exact sequence

$$
0 \rightarrow K \rightarrow \stackrel{f}{\rightarrow} N \rightarrow 0
$$

of (finitely generated) left $\boldsymbol{R}$-modules yields an exact sequence

$$
0 \rightarrow N^{*} \stackrel{f^{*}}{\rightarrow} M^{*} \rightarrow \text { Coker } f^{*} \rightarrow 0
$$

of (finitely generated) torsionless right $R$-modules, since Coker $f^{*}$ embeds in $K^{*}$. Thus (d) implies that $f^{* *}$ is epic.

REMARKS. (1) Over hereditary artinian (noetherian) rings, (finitely generated) torsionless modules are projective. Thus ()$^{* *}$ preserves epimorphisms (of finitely generated modules) over any such ring.

(2) If $R$ is an indecomposable serial (i.e. generalized uniserial) ring that is neither hereditary nor QF, then $R$ has a Kupisch series $R e_{1}, \ldots, R e_{n}$ such that for some $1<i<n, R e_{i-1} \cong J e_{i}$ and $1<c\left(R e_{i+1}\right)<c\left(R e_{i}\right)$, where $c(M)$ denotes composition length and $J$ is the radical of $R$. If $k=c\left(R e_{i+1}\right)-1$, then $R e_{i} / J^{k} e_{i} \simeq J e_{i+1}$ is torsionless and there is a monomorphism $J^{k} e_{i} \rightarrow R e_{i-1}$ (see [4]). This clearly cannot extend to a map into $R$, so condition (d) of Theorem 4 does not hold. Thus we see that over an indecomposable serial ring ( )** preserves epimorphisms (of finitely generated modules) if and only if the ring is hereditary or QF. 
(3) Let $R$ be the ring of $4 \times 4$ upper triangular matrices of the form

$$
\left[\begin{array}{llll}
a & x & y & z \\
& b & 0 & u \\
& & c & v \\
0 & & & a
\end{array}\right]
$$

with entries in a field $F$. Let $e$ be the matrix with $a=1$ and zeros elsewhere. Then every indecomposable left (right) $R$-module is isomorphic to a submodule of a quotient module of $R e$ (resp. eR) (see [10], for example), and $R e / J e$ and $J e$ (resp. $e R / e J$ and $e J)$ are the only nonprojective torsionless modules among them. Thus, upon checking that $(R e / J e)^{*} \simeq e J,(J e)^{*} \simeq e R / e J,(e R / e J)^{*} \simeq J e$, and $(e J)^{*} \simeq$ $\mathrm{Re} / \mathrm{Je}$ we can apply Theorem 4(c) to show that ( )** preserves epimorphisms of finitely generated $R$-modules even though $R$ is neither hereditary nor QF.

\section{REFERENCES}

0. F. W. Anderson and K. R. Fuller, Rings and categories of modules, Springer-Verlag, New York, Heidelberg and Berlin, 1974.

1. S. U. Chase, Direct products of modules, Trans. Amer. Math. Soc. 97 (1960), 457-473.

2. R. R. Colby and E. A. Rutter, Jr., Generalizations of QF-3 algebras, Trans. Amer. Math. Soc. 153 (1971), 371-386.

3. C. Faith, Rings with ascending chain conditions on annihilators, Nagoya Math. J. 27 (1966), 179-191.

4. K. R. Fuller, Generalized uniserial rings and their Kupisch series, Math. Z. 106 (1968), 248-260.

5. __ On indecompasable injectives over artinian rings, Pacific J. Math. 29 (1969), 115-135.

6. K. Morita, Duality in QF-3 rings, Math. Z. 108 (1969), 237-252.

7. B. J. Mueller, The classification of algebras by dominant dimension, Canad. J. Math. 20 (1968), 398-409.

8. E. A. Rutter, Jr., Two characterizations of quasi-Frobenius rings, Pacific J. Math. 30 (1969), 777-784.

9. H. Tachikawa, Quasi Frobenius rings and generalizations, Lecture Notes in Math., vol. 351, Springer-Verlag, Berlin and New York, 1973.

10. On algebras of which every indecomposable representation has an irreducible one as the top or the bottom Loewy constituent, Math. Z. 75 (1961), 215-227.

Department of Mathematics, Universtty of HawaII, Honolulu, Hawail 96822

Department of Mathematics, University of Iowa, Iowa City, Iowa 52242 